\title{
Multi-Parameter Low-Power Sensor Network for Aquatic Research
}

\author{
L. Wahn', T. Gentz², H.K. Trieu1, M. Schlüter', J. Müller ${ }^{1}$ \\ ${ }^{1}$ Institute for Microsystems Technology, TU Hamburg-Harburg, Germany \\ lennart.wahn@tuhh.de \\ ${ }^{2}$ Alfred Wegener Institute for Polar and Marine Research, Bremerhaven, Germany
}

\begin{abstract}
:
A new approach was developed for an underwater sensor network to simultaneously measure multiple environmental parameters, relevant for the research of aquatic systems. In marine science spatial and time-dependent variations of base parameters are of significant interest regarding environmental influenced biodiversity in shelf areas. For example, the influence of submarine groundwater discharge (SGD) on marine organisms are part of today's scientific research [1]. The sensor network consist of a fault-tolerant CAN-BUS topology and is designed to simultaneously measure at up to 120 different locations over a maximum range of $1 \mathrm{~km}$ providing excellent spatial resolution. First field measurements were performed and it has been shown that it is feasible to map multiple environmental parameters with good temporal and spatial resolution.
\end{abstract}

Key words: underwater sensor network, submarine groundwater discharge, CAN-BUS

\section{Introduction}

Many studies indicate that submarine groundwater discharge (SGD) is not negligible in terms of freshwater exchange in coastal areas and have a high impact on benthic habitats, and nutrient release from sediments into the water column [2]. In some areas it even exceeds the magnitude of river-borne chemical fluxes [3]. The different temperatures and salinities of SGD induce a regional change in the aquatic biotope. Also the exchange of water between land and sea and therefore effects from chemical flux and water pollution can be observed and directly compared to unaffected areas. Most sensors used in aquatic research are single units making it impossible to simultaneously measure large areas in a short period of time.

\section{Technical Realisation}

The actual sensor setup allows online, realtime, and in situ measurements of temperature, light intensity, and pressure. The sensor network is designed to simultaneously measure at up to 120 different locations with varying distances from the coast over a maximum range of $1 \mathrm{~km}$ providing excellent spatial resolution. Its low-power concept enables long term measurements including adjustable sample rates for fast and slow changing parameters. The modular concept of the network offers high upgradability with additional sensors i.e. for impedance analyses. The network consists of a flexible number of nodes in a fault-tolerant CAN-BUS topology which are molded in epoxy resin for underwater application. In automotive application CAN-BUS is already well established as it has been designed for especially harsh environments. With differential signals, cross-wire, and loss of ground protection an operation in saltwater with unknown conditions is possible [4]. The obtained data can be stored on the home node in a NVRAM or transmitted over Sub- $1 \mathrm{GHz}$ RF. A maximum sample rate of 2 samples per second for every sensor node and sensor is feasible. Each node can be supplied with voltages between 5 and $24 \mathrm{~V}$ utilizing several different power supplies, i.e. batteries or solar modules. Communication and periphery is controlled by a MSP430 $4 \mathrm{M}$ microcontroller (Texas Instrument $($ ) ) for maximum power savings. The CAN-BUS communication is done through a CAN transceiver SN65HVD234 from Texas Instrument $\Omega$ and a CAN controller MCP2515 Microchip $\circledast$. The CAN controller handles the transmitting and receiving of messages over the CAN-BUS. The CAN transceiver drives the CAN-BUS at a voltage level of $3.3 \mathrm{~V}$ and protects the circuit. The 
different functionalities of a home and sensor node are depicted in figure 1.

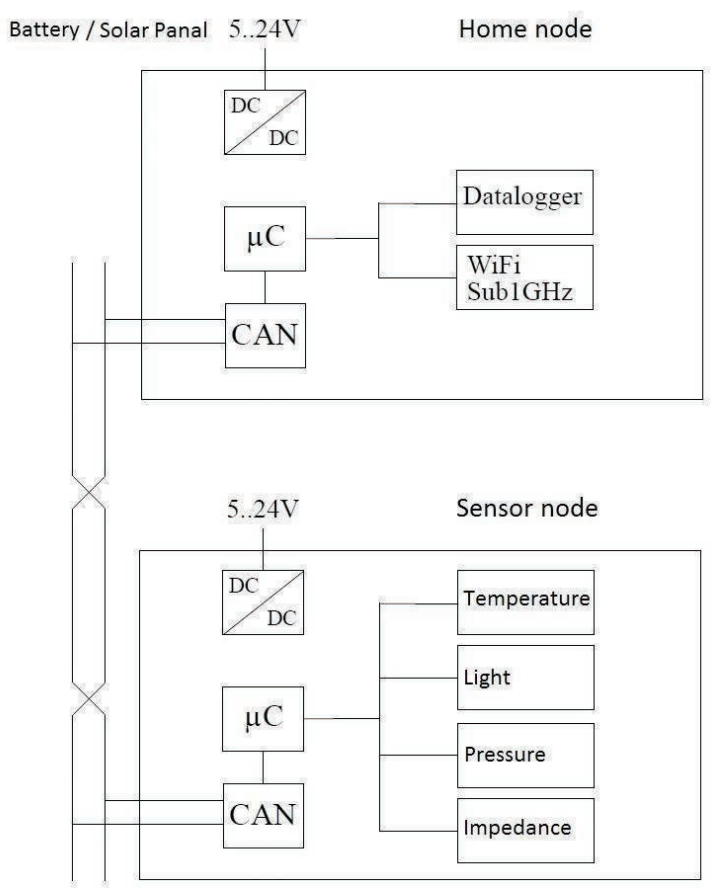

Fig. 1. Functional blocks of a home and sensor node

The DC/DC converters provide the circuit with $5 \mathrm{~V}$ and $3.3 \mathrm{~V}$ for maximum flexibility for different sensor concepts. In figure 2 one sensor node with four connections for power supply and CAN-BUS on top of an adapter PCB where a CAT5 cable is connected is visible.

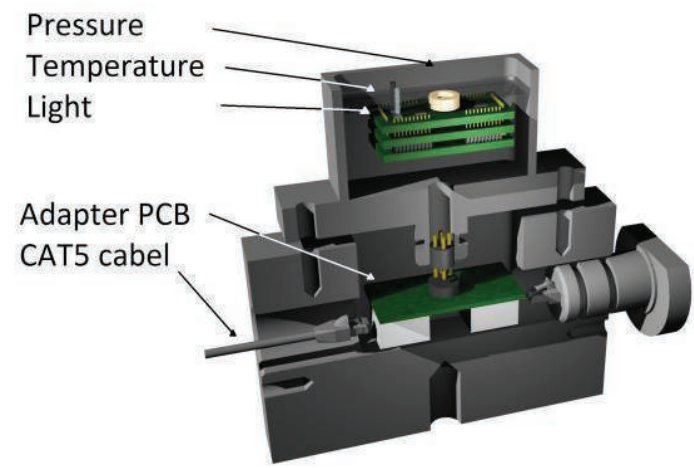

Fig. 2. Schematic view of the sensor node on adapter block

The sensor node is molded in epoxy resin and the pressure sensitive membrane is left free. On the temperature sensor a metal rod is glued to provide a good heat transfer from the water to the sensor. On the light sensor a Plexiglas rod is glued for light transmission. The enclosure of the sensor node is made out of polyoxymethylen to provide higher stability against salt water. The adapter for CAT 5 cable connects the CAN-BUS and the power line over a four pin connector to the sensor PCB. The connection from one sensor node to another is done through a standard CAT 5 cable. The RJ45 connector is enhanced with a polyoxymethylen connector to protect the adapter PCB from water. On the bottom of the sensor node a thread is provided and a staff can be attached to anchor the sensor node on the sea ground. Figure 3 shows the setup for first test measurements. The home node is placed in the aluminium pressure housing and can be accessed over a SubCon ${ }^{\circledR}$ connector for measurement setup and downloading measurement data. Over the same connector a Sub1Ghz WiFi module can be attached for wireless transfer of the measurement data to a PC.

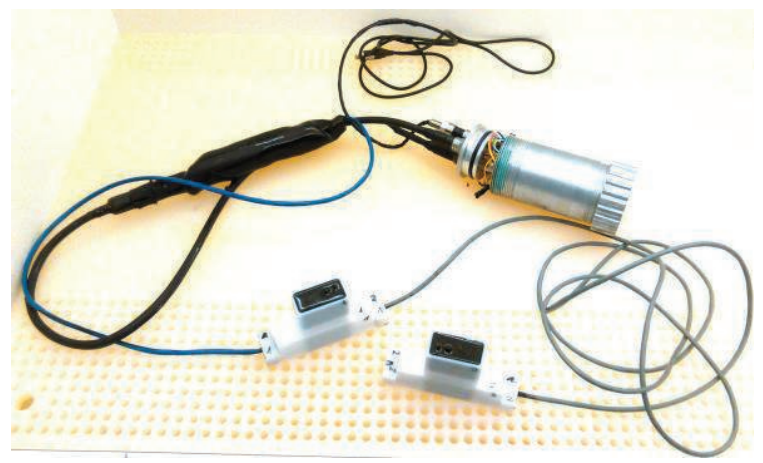

Fig. 3. Measurement setup with two nodes and a home node in a metal enclosure

The wireless communication was tested in the laboratory with four sensor nodes and one home node. To the home node a Sub $1 \mathrm{GHz}$ wireless module from Texas Instrument $\AA$ was attached. Each second one measurement each seconds was performed and transmitted to a PC. The complete network has been powered by a $9 \mathrm{~V}$ block battery. Figure 3 shows the light intensity of four sensor nodes. The decrease of light intensity of Node 1 and Node 2 is due to molded in epoxy resin compared to Node 3 and Node 4. Data had been collected over a 90 minute time period. The measurement curves resemble each other and different light intensities are due to different exposure to the light in the laboratory. 


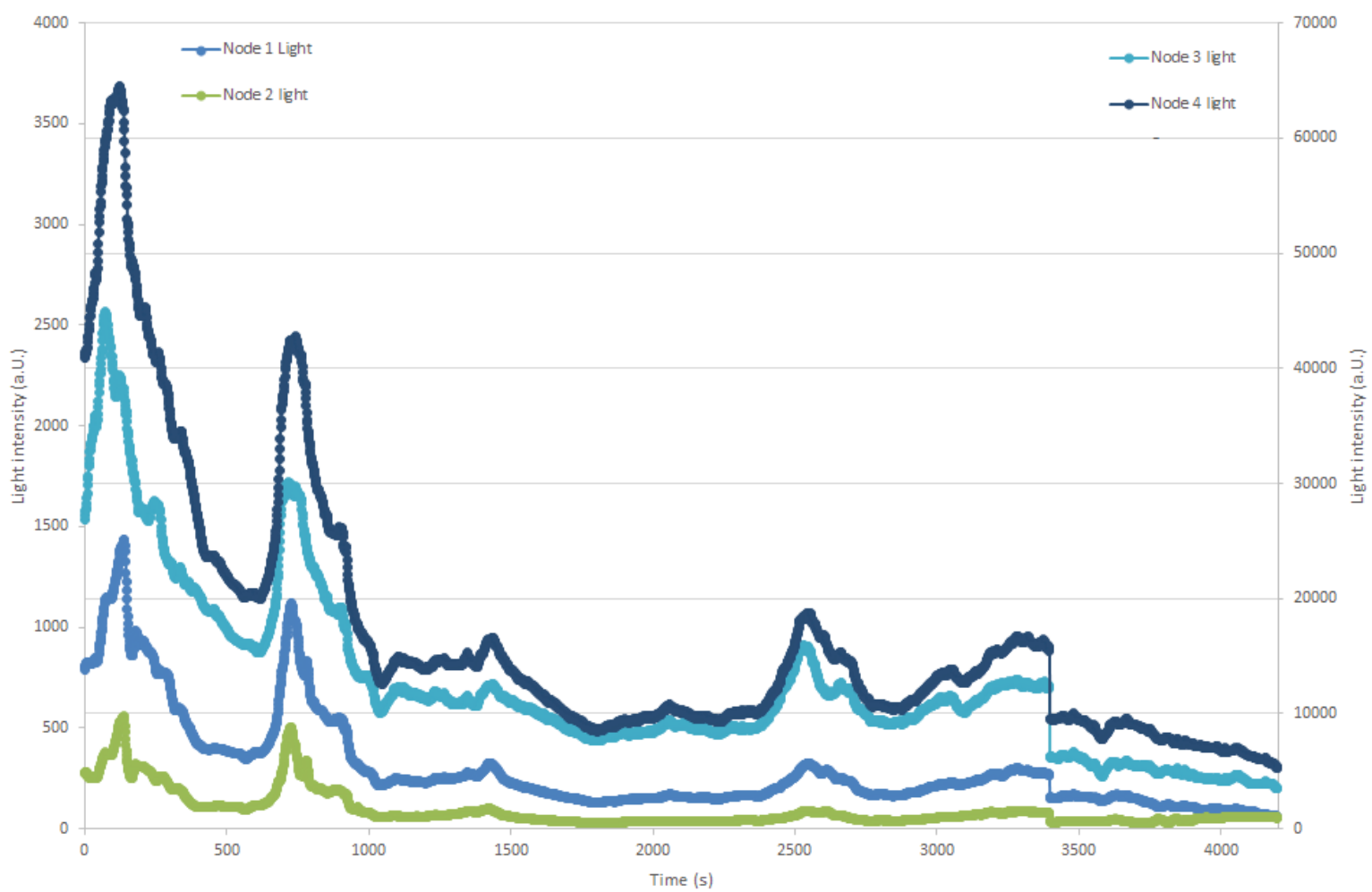

Fig. 4. Light intensity of four sensor nodes

\section{Field Measurement}

First field measurements were performed in the North Sea near Cuxhaven, Germany, with two nodes for temperature and light intensity measurements and one node responsible for initialization and data logging. Measurements were initialized and stored over a period of 150 minutes with one sample per minute. Figure 5 shows light intensity and temperature over time.

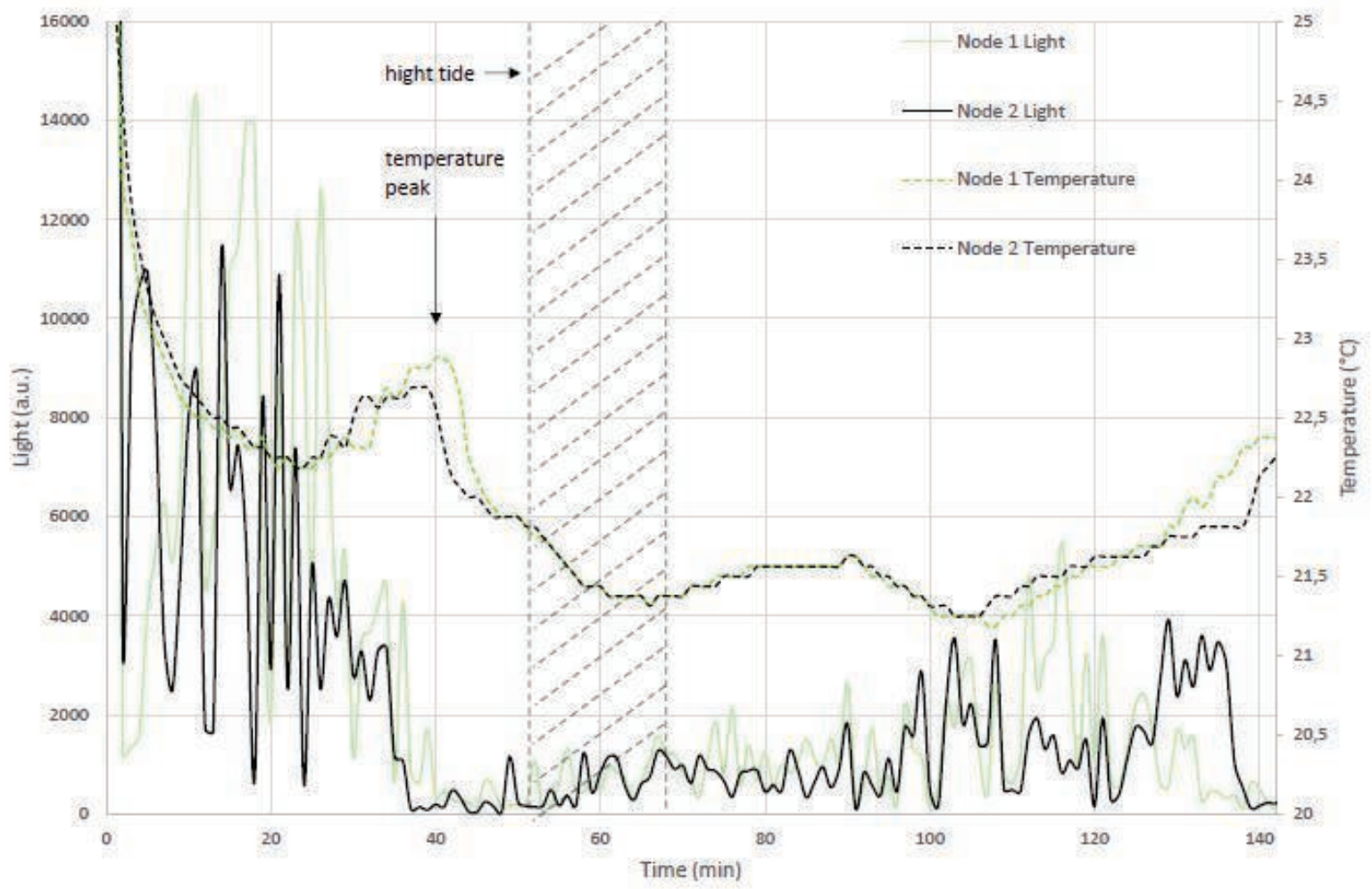

Fig. 5. Field measurement of temperature and light intensity near Cuxhaven, Germany on a cloudy midday at 20.06.2014. 
High tide had been reached after 40 to 60 minutes. During the first 30 minutes of the measurement direct sunshine heated up the environment resulting in a temperature peak around 40 minutes. Afterwards it was mostly clouded. After high tide water depth decreased light intensity increased. Just before high tide the temperature curve shows a delay between node 1 and 2 in such a way that the temperature peak arrives later at node 1. At the end of the measurement this behaviour is inverted. This phenomenon is explained with the direction of the water current during this measurement. Node 1 was placed closer to the coast than node 2 and before high tide the water moves toward the coast and afterwards away. Figure 6 shows a schematic view of the sensor network with two sensor nodes.

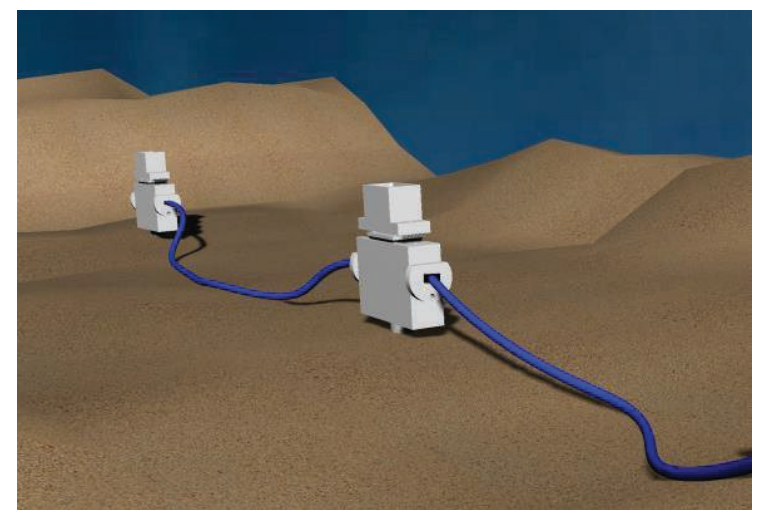

Fig. 6. Schematic view of the sensor network

\section{Conclusion}

It has been shown that it is feasible to map multiple environmental parameters with good temporal and spatial resolution. The sensor network can operate with multiple sensor nodes over several hours and can store or directly transmit the data. Wireless data transfer was performed under laboratory condition with four sensor nodes including temperature, light and pressure sensors. The same arrangement was capable to measure and store data once a minute over more than 4 days with a $250 \mathrm{mAh}$ $9 \mathrm{~V}$ block battery.

\section{References}

[1] L. Kotwicki, K. Grzelak, M. Czub, O. Dellwig, T. Gentz, B. Szymczycha and M. Böttcher, Journal of Marine Systems 129, 118-126 (2013); doi: 10.1016/j.jmarsys.2013.06.009

[2] M. Schlüter, E. J. Sauter, C. E. Andersen, H. Dahlgaard, P. R. Dando, Limnol. Oceanogr., 49(1), 2004, 157-167

[3] W.C. Burnett, H. Bokuniewicz, M. Huettel, W.S. Moore, M. Taniguchi, Biogeochemistry, 66 (2003), pp. 3-33; doi: 10.1023/B:BIOG.0000006066.21240.53

[1] [4] Texas Instrument, 3.3-V CAN TRANSCEIVERS SN65HVD234, (2008); 